\title{
Overmedicalization of Pregnancy and Childbirth
}

\author{
Fahimeh Ranjbar $^{1}$, Maryam Gharacheh $^{1^{*}}{ }^{(\mathbb{D}}$, AbouAli Vedadhir $^{2}$
}

\section{Dear Editor,}

Medicalization is regarded as a form of social control and a process through which problems or non-medical experiences are defined and managed as medical problems or diseases. Furthermore, medicine is increasingly dominating the everyday life of individuals (1), thereby resulting in the social acceptance of the medicalized form of human experiences. Via attaching a disease label to natural phenomena, medical professionals expand the scope of their authority, regardless of whether they have the capacity to effectively manage these phenomena (2). The current paper did not intend to undervalue medical achievements, especially in the area of maternity care, but rather, to depict the overmedicalization of the experience of pregnancy and childbirth.

Within the past two decades, medicalization has altered the concept of pregnancy and mothering experience. Now, pregnancy and childbirth are not viewed as natural events but as medical events (3). Moreover, the increased rates of screening and monitoring of pregnancy to reduce the risks of maternal and fetal morbidity and mortality have led to the overmedicalization of pregnancy and childbirth (4). In other words, it seems the risks associated with pregnancy and childbirth are magnified, thereby increasing the concerns of women regarding themselves and their neonates. In addition, "expecting trouble" has currently become the hallmark of prenatal care, leading to an exaggerated concern in women. I this view, it is not surprising that medical interventions during pregnancy and childbirth have resulted in the increased rates of cesarean section along with maternal mortality (5).

Medicalization of pregnancy has even affected the natural attachment between the mother and fetus. Accordingly, delayed maternal-fetal attachment could adversely influence the early development of pregnancy (6). Furthermore, medicalization has altered the individuals' perceptions of the professional skills of midwives, and in this regard, obstetricians have replaced midwives in normal deliveries (7). Although a teamwork between midwives and obstetricians is required for the provision of maternity care, growing evidence suggests that the involvement of obstetrician is unnecessary in the management of low-risk pregnancies, as it leads to increased medical interventions compared to the midwifery models of care (8). In low-risk pregnancies, the development of midwifery-led care has been reported to be more cost-efficient in the reduction of maternal mortality rate compared to the current care models, as well as increasing access to antenatal care. However, safety standards should not be compromised in any model of maternity care, as this would not be justified at any cost (9).

Despite our perception of the mother's right to make informed medical decisions, the concept of choice is not real in the medical model of motherhood, since women are treated like children and therefore "the passive recipients" of health care, whose individual identity and autonomy is neglected due to their loss of control over their body (10). In contrast to the nature's plan that is based on the confidence and competence of women, standard prenatal care and medicalized labor lead to substantial fear, concern, and uncertainty in expectant mothers (11).

Considering the abovementioned, some medical interventions in pregnancy and childbirth are unnecessary and devalue the personal experiences and abilities of women in this regard (12). Therefore, the demedicalizing approach has recently been proposed and applied in the area of maternity care, playing a pivotal role in the new clinical guidelines. This idea has further encouraged the women's right to choose their preferred care during pregnancy and childbirth. In this regard, the World Health Organization guideline on antenatal care is focused on reducing the number of prenatal visits and standard tests, as well as on the physiological process of pregnancy and childbirth.

In Iran, the rate of cesarean section has been estimated to be $50 \%$ (13) and the unpleasant experiences of mothers in vaginal delivery (14) are the evidence of the overmedicalization of pregnancy and childbirth. Most mothers in Iran prefer medicalized childbirth under the supervision of obstetricians. The selection of caesarean delivery within the past three decades has led

Received 13 September 2016, Accepted 17 February 2017, Available online 15 March 2017

${ }^{1}$ Nursing Care Research Centre, Iran University of Medical Sciences, Tehran, Iran. ${ }^{2}$ Department of Anthropology, Faculty of Social Sciences,

University of Tehran, Tehran, Iran

*Corresponding Author: Maryam Gharacheh, Tel: +98 2188671613, Email: gharacheh.m @gmail.com 
to the increased authority of medicine, which in turn has diminished the role of midwives in vaginal delivery (15). The expansion of medicalization in this area (16) has caused significant challenges in the implementation of "promotion of natural childbirth" in line with the Health Reform Plan, requiring further investigations in the disciplines of sociology, policy making, and health economics.

\section{Conflict of Interests}

Authors declare that they have no conflict of interests.

\section{Ethical Issues}

Not applicable.

\section{Financial Support}

None.

\section{References}

1. Coser RL. Socio-Medical Inquiries: Recollections, Reflections, and Reconsiderations. JAMA. 1985;253(5):695695. doi:10.1001/jama.1985.03350290101043

2. Ghazi Tabatabaie M, Vedadhir AA. Surrogacy: Medicalization of Motherhood. J Reprod Infertil. 2008;9(2):144-164.

3. Prosen M, Tavcar Krajnc M. Sociological conceptualization of the medicalization of pregnancy and childbirth: the implications in Slovenia. Rev Sociol. 2013;43(3):251-272. doi:10.5613/rzs.43.3.3

4. Fordyce L. Accounting for fetal death: vital statistics and the medicalization of pregnancy in the United States. Soc Sci Med. 2013;92:124-131. doi:10.1016/j. socscimed.2013.05.024

5. Lothian JA. The journey of becoming a mother. J Perinat Educ. 2008;17(4):43-47. doi:10.1624/105812408x364071

6. Rowe R, Puddicombe D, Hockley C, Redshaw M. Offer and uptake of prenatal screening for Down syndrome in women from different social and ethnic backgrounds. Prenat Diagn. 2008;28(13):1245-1250. doi:10.1002/pd.2125
7. Barker KK. A ship upon a stormy sea: the medicalization of pregnancy. Soc Sci Med. 1998;47(8):1067-1076. doi:10.1016/S0277-9536(98)00155-5

8. Schroeder L, Petrou S, Patel N, et al. Birthplace costeffectiveness analysis of planned place of birth: individual level analysis Birthplace in England research programme. Southampton: HMSO; 2011.

9. Ryan P, Revill P, Devane D, Normand C. An assessment of the cost-effectiveness of midwife-led care in the United Kingdom. Midwifery. 2013;29(4):368-376. doi:10.1016/j. midw.2012.02.005

10. Nicol M. Vulnerability of first-time expectant mothers during ultrasound scans: an evaluation of the external pressures that influence the process of informed choice. Health Care Women Int. 2007;28(6):525-533. doi:10.1080/07399330701334281

11. Lothian JA. Safe, healthy birth: what every pregnant woman needs to know. J Perinat Educ. 2009;18(3):48-54. doi:10.1624/105812409x461225

12. Johnson C. The political "nature" of pregnancy and childbirth. Can J Polit Sci. 2008;41(4):889-913. doi:10.1017/ S0008423908081079

13. Shahshahan Z, Heshmati B, Akbari M, Sabet F. Caesarean section in Iran. Lancet. 2016;388(10039):29-30. doi:10.1016/ s0140-6736(16)30899-6

14. Rahnama P, Mohammadi K, Montazeri A. Salient beliefs towards vaginal delivery in pregnant women: A qualitative study from Iran. Reprod Health. 2016;13:7. doi:10.1186/ s12978-016-0120-5

15. Sedigh Mobarakabadi S, Mirzaei Najmabadi K, Ghazi Tabatabaie M. Ambivalence towards childbirth in a medicalized context: a qualitative inquiry among Iranian mothers. Iran Red Crescent Med J. 2015;17(3):e24262. doi:10.5812/ircmj. 24262

16. Sedigh Mobarakabadi S, Mirzaei Najmabadi K, Ghazi Tabatabaie M, Esmaily H. Predictors of mode of childbirth based on medicalized maternal care: a cross-sectional study. Iran Red Crescent Med J. 2017;19(2):e25073. doi:10.5812/ ircmj.25073

(c) 2019 The Author (s); This is an open-access article distributed under the terms of the Creative Commons Attribution License (http://creativecommons.org/licenses/by/4.0), which permits unrestricted use, distribution, and reproduction in any medium, provided the original work is properly cited. 